\title{
Optimal sedative dose of propofol to start MRI in children with cerebral palsy
}

\author{
Eun Jung Kim ${ }^{1}$, Youn Yi Jo ${ }^{2}$, and Hae Keum Kil ${ }^{1}$ \\ Department of Anesthesiology and Pain Medicine, ${ }^{1}$ Yonsei University College of Medicine, Seoul, ${ }^{2}$ Gachon University of Medicine \\ and Science Gil Medical Center, Incheon, Korea
}

Background: This study was designed to determine the optimal sedative dose of propofol to start brain magnetic resonance imaging (MRI) in children with cerebral palsy (CP).

Methods: Twenty children, aged $0.5-5$ years, were administered propofol to achieve a University of Michigan Sedation Scale (UMSS) score $\geq 3$ in the MRI room. The proper dose of propofol was determined using the up-anddown method.

Results: The ED50 and ED95 for successful sedation with a UMSS $\geq 3$ were $2.07 \mathrm{mg} / \mathrm{kg}$ (95\% CI 1.69-2.56) and 2.69 $\mathrm{mg} / \mathrm{kg}$ (95\% CI 2.35-5.59). Respiratory events occurred in 5 patients and were resolved with neck extension, chin lift, or transient respiratory assistance with successful sedation.

Conclusions: Low dose propofol can safely facilitate the initiation of MRI in children with CP. (Korean J Anesthesiol $2011 ; 61: 216-219)$

Key Words: Cerebral palsy, MRI, Propofol.

\section{Introduction}

Cerebral palsy (CP) is the general term for a group of nonprogressive but often changing motor impairment syndromes secondary to early brain development disorders. Clinical features may vary from mild monoplegia with normal intellect to severe body spasticity and mental retardation. Two neuroimaging studies reported that $70 \%$ and $90 \%$ of children with CP showed brain abnormalities [1,2]. Thus, MRI is a part of the routine investigation performed for a precise evaluation of children with CP. MRI requires complete immobility for accurate imaging during examination. Particularly in CP with spasticity or athetosis, deep sedation is required to control behaviour [3].

Propofol is a popular hypnotic agent for pediatric sedation because of its rapid onset, effective anaesthesia, rapid and complete recovery, and prevention of nausea and vomiting [4-7]. Children with CP have shown a drug response that is different from normal children, possibly related to an interaction with various oral medications such as baclofen, tizanidine,

Received: December 2, 2010. Revised: 1st, January 12, 2011; 2nd, January 26, 2011. Accepted: February $11,2011$.

Corresponding author: Youn Yi Jo, M.D., Department of Anesthesiology and Pain Medicine, Gachon University of Medicine and Sciene Gil Medical Center, 1198, Guwol-dong, Namdong-gu, Incheon 405-760, Korea. Tel: 82-32-460-3636, Fax: 82-32-469-6319, E-mail: endless37@hanmail.net @ This is an open-access article distributed under the terms of the Creative Commons Attribution Non-Commercial License (http:// creativecommons.org/licenses/by-nc/3.0/), which permits unrestricted non-commercial use, distribution, and reproduction in any medium, provided the original work is properly cited. 
diazepam, dantrolene, or anticonvulsant drugs [8,9].

We hypothesized that $\mathrm{CP}$ children may require less propofol than non-CP children for proper sedation during MRI. The aim of this study is to establish the optimal induction dose of propofol for deep sedation to start MRI for less than $30 \mathrm{~min}$ in children with CP.

\section{Materials and Methods}

This study was approved by the institutional review board of our hospital and registered with clinicaltrial.gov. Written informed consent was obtained from all eligible participants' parents for this study. Children with obstructive sleep apnea, anticipated difficult airway, evidence of cardiopulmonary disease, or allergic history to propofol were excluded from this study.

Inclusion criteria were ASA status I or II with CP, aged 6 months to 5 years, scheduled for brain MRI under sedation. No child was premedicated. After intravenous (IV) access was established by an expert nurse in the preparation room, children were transferred to the MRI room with their parents. After obtaining an electrocardiogram and measuring noninvasive blood pressure and pulse oximetry, a predetermined dosage of propofol mixed with lidocaine $2 \mathrm{mg} / \mathrm{ml}$ was administered over $30 \mathrm{~s}$ through the IV. An anesthesiologist blinded to the study protocol assessed the sedation level using a University of Michigan Sedation Scale (UMSS) (Table 1). If the child showed a UMSS score $\geq 3$ after $1 \mathrm{~min}$ of propofol i.v. and showed no movement at the beginning of MRI examination, the case was considered successful sedation. If the child showed a score $<3$, required a titrated additional dose of propofol to deepen the sedation, then the case was considered a failed case. The modified Dixon's up-and-down method by Paul and Fisher $[10,11]$ was used to determine the propofol ED50 for appropriate sedation to start the MRI examination. The up-anddown method required six independent pairs of consecutive subjects in which a success score followed by a failure score was obtained. The initial propofol dose was $2 \mathrm{mg} / \mathrm{kg}$. If the sedation failed with the initial dose, propofol was increased by $0.5 \mathrm{mg} /$

Table 1. The University of Michigan Sedation Scale (UMSS)

\begin{tabular}{cc}
\hline Value & Patient state \\
\hline 0 & Awake and alert \\
1 & Minimally sedated: tired/sleepy, appropriate response to \\
& verbal conversation, and/or sound \\
2 & Moderately sedated: somnolent/sleeping, easily aroused \\
& with light tactile stimulation or a simple verbal command \\
3 & $\begin{array}{c}\text { Deeply sedated: deep sleep, aroused only with significant } \\
\text { physical stimulation }\end{array}$ \\
4 & Unarousable \\
\hline
\end{tabular}

www.ekja.org $\mathrm{kg}$ for the next child. If the sedation was successful, the next patient received a propofol dose decreased by $0.5 \mathrm{mg} / \mathrm{kg}$. The ED50 of propofol for a UMSS score $>3$ was defined by averaging the mid-point dose of all independent pairs of patients involving a crossover. Therefore, the number of patients was not determined prior to this study.

A soft roll was placed under the patient's shoulder to slightly extend the neck. Supplemental oxygen was given by a transparent face mask at a flow rate of $5 \mathrm{~L} / \mathrm{min}$. End-expiratory carbon dioxide $\left(\mathrm{ETCO}_{2}\right)$ was monitored via a nasal prong. Adequate spontaneous respiration without an artificial airway was established before examination. ECG, peripheral oxygen saturation $\left(\mathrm{SpO}_{2}\right)$, and $\mathrm{ETCO}_{2}$ were monitored continuously, and noninvasive blood pressure was measured at 5-min intervals throughout the procedure. In cases of inadequate sedation during MRI, an additional $0.5 \mathrm{mg} / \mathrm{kg}$ of propofol was administered intermittently.

After MRI examination, the patient was transferred to the sedation room with supplemental oxygen and pulse oximetry. During recovery, a blinded observer evaluated side effects such as oxygen desaturation, emergence agitation, postoperative nausea, and vomiting. When the children met the discharge criteria, they were discharged from the recovery room. A telephone interview was performed to determine the presence of any abnormal behaviour, fever, nausea, or vomiting during the first 24 hours.

Statistical analysis was performed using PASW Statistics $18^{\circledR}$ (SPSS Inc, Chicago, IL, USA). Data are expressed as the mean \pm SD or the number of patients (\%). The ED50 of propofol for tracheal intubation was defined by averaging the mid-point dose of all independent pairs of patients involving a crossover. The "up-and-down" data were also analyzed by a probit analysis, which enabled us to derive the mean propofol dose with a $95 \%$ confidence interval. A P value $<0.05$ was considered significant.

\section{Results}

One patient was excluded from data analysis because ketamine was used during examination. The patients' characteristics and outcomes during MRI scanning are presented in Table 2. Four children had ataxia, seven had athetosis, and nine had a spastic type of CP. Four of the 20 children had taken anticonvulsants (carbamazepine, clonazepam, sodium valproate, or topiramate), and one had used anticholinergic drug (oxybutynin). All children had abnormal results in official readings of their brain MRI.

The sequence of successful and unsuccessful induction of deep sedation is shown in Fig. 1. The ED50 and ED95 for successful sedation with UMSS $\geq 3$ were $2.07 \mathrm{mg} / \mathrm{kg}$ (95\% CI 
Table 2. Patient Characteristics and Outcome Measures

\begin{tabular}{lc}
\hline \multicolumn{1}{c}{$\mathrm{N}$} & 20 \\
\hline Age (months) & $28.7 \pm 17.8$ \\
Weight (kg) & $10.3 \pm 4.6$ \\
Height (cm) & $82.6 \pm 10.8$ \\
Sex (M/F) & $16 / 4$ \\
Seizure medication & $4 / 20$ \\
MRI scanning time (min) & $26.3 \pm 4.0$ \\
Recovery time (min) & $19.8 \pm 9.4$ \\
Children requiring additional propofol (n) & 10 \\
Total dose of propofol (mg/kg) & $3.2 \pm 0.9$ \\
\hline
\end{tabular}

Values are the mean \pm SD, the median (range), or the number of patients.

$1.69-2.56)$ and $2.69 \mathrm{mg} / \mathrm{kg}$ (95\% CI 2.35-5.59), respectively. Immediately after propofol injection, respiratory events (desaturation $<95 \%$ and partial airway obstruction) occurred in five patients with successful sedation $\left(\mathrm{SpO}_{2}<95 \%\right.$ in four and $<85 \%$ in one), which were promptly resolved with a neck extension, chin lift, or transient respiratory assistance. No other adverse events such as hypotension, bradycardia, or arrhythmia were observed during the MRI examination. All scheduled MRI examinations were successfully completed within 30 minutes. In 10 children, additional propofol was administered. No one showed emergence agitation, abnormal behaviour, fever, or nausea and vomiting during recovery after sedation or in the first $24 \mathrm{~h}$ after discharge.

\section{Discussion}

The goals of sedation in children for diagnostic and therapeutic procedures are to (1) guard their patients' safety and welfare; (2) minimize physical discomfort and pain; (3) control anxiety, minimize psychological trauma, and maximize the potential for amnesia; (4) control behaviour and or movement to allow safe completion of the procedure; and (5) return the patient to a state in which safe discharge from medical supervision, as determined by recognized criteria, is possible [12]. These goals can best be achieved by selecting the lowest dose of a drug with the highest therapeutic index for the procedure.

Children with CP may demonstrate a decreased MAC of volatile anaesthetics when compared to healthy children (e.g. the MAC of halothane has been shown to be $20 \%$ lower) [8]. The MAC of halothane may be further lowered in children with $\mathrm{CP}$ taking anticonvulsants for seizure disorders [8]. In a study using BIS, the propofol dosage for a BIS value of 35-45 was also lower in children with CP than in healthy children $(3.29 \pm 0.5 \mathrm{mg} / \mathrm{kg}$ vs. $3.6 \pm 0.9 \mathrm{mg} / \mathrm{kg}$ ) [9]. However, no difference was found in their study between the propofol dosages of CP children using seizure medication and those who were not. We did not exclude children who had taken seizure medication, but we could not

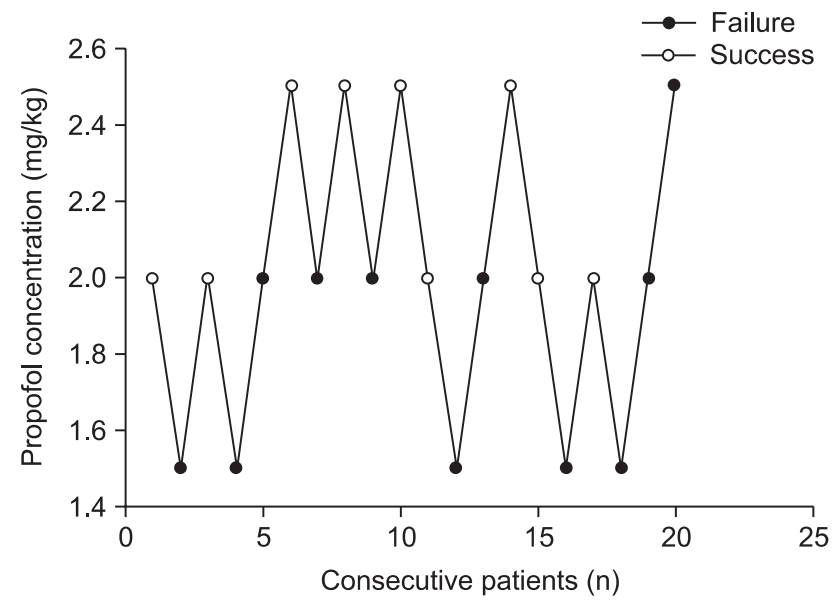

Fig. 1. Responses of the 20 consecutive children for whom sedation for MRI scanning was attempted and the propofol concentration. Each patient's data are represented by a circle.

verify whether the seizure medication had any affect on the propofol dosage for deep sedation. Our results showed that the ED50 and ED95 of propofol for successful deep sedation to perform MRI were $2.07 \mathrm{mg} / \mathrm{kg}$ (95\% CI 1.69-2.56) and 2.69 $\mathrm{mg} / \mathrm{kg}$ (95\% CI 2.35-5.95), respectively, in children with CP. The mean total dose of propofol was $3.2 \pm 0.9 \mathrm{mg} / \mathrm{kg}$, which was higher than the $2.4 \pm 0.7 \mathrm{mg} / \mathrm{kg}$ results of a study performed in children who underwent MRI for less than 30 min by Cho et al. [13]. Cho et al. enrolled children with various cause (delayed development, seizure, cerebral palsy, brain tumor, etc.in their study, while we enrolled children with CP. For this study, we hypothesized that $\mathrm{CP}$ children may require less propofol than non-CP children for proper sedation during MRI. In our study, 16 children had behavioural disturbances ( 7 were athetotic and 9 were spastic). We obtained results that were the opposite of our hypothesis for the propofol dosage needed to get a sedation level $\geq$ UMSS 3 . Considering these results, we can presume that more propofol was required to get proper sedation in children with behavioural disturbances, while a lower dose of propofol was required in children with $\mathrm{CP}$ than in children without $\mathrm{CP}$ during induction of anesthesia [9]. Sometimes, propofol alone does not seem to suppress unintentional movements during sedation, as was the case in a study where a large dose of propofol (3.9 mg/kg, 1.8-6.4) was used to induce deep sedation without unintentional movements during MRI examination in children [14]. However, a large dosage of propofol can induce severe respiratory depression, and children may quickly move from one level of sedation to another. Furthermore, MRIcompatible BIS or other monitors for sedation assessment are not yet available in practice. Therefore, the dosage of propofol must be carefully titrated with close observational scaling of the sedation level during MRI. In our study, the sedation 
level was assessed by an anesthesiologist well-experienced at using the UMSS. We defined successful deep sedation as when the children showed a UMSS $\geq 3$, in deep sleep, aroused only with significant physical stimulation. A UMSS $\geq 3$ may be deep sedation, inducing respiratory depression. In our study, $\mathrm{SpO}_{2}$ was decreased $(<95 \%)$ in 5 children with successful deep sedation $(5 / 20,25 \%)$ after propofol injection, but the problems were soon resolved with proper management.

To determine the ED95 of the drug, logistic regression is commonly used. However, the up-and-down approach for analysis of quantal data may yield incorrect estimates. We used the modified Dixon's up-and-down method, with a modification by Paul and Fisher [11], aiming for 6 crossovers in order to decrease the likelihood of reporting an inaccurate estimate. Nevertheless, the up-and-down method does not provide reliable insight into the upper tail of the distribution, and the assumption that the dose-response curve of the drug is wellfitted by a symmetric logistic curve remains unverified [15]. This is a limitation of our study in defining the ED95 of propofol for sedation of children with CP.

In summary, the ED50 and ED95 of propofol were $2.07 \mathrm{mg} /$ $\mathrm{kg}$ (95\% CI $1.69-2.56)$ and $2.69 \mathrm{mg} / \mathrm{kg}$ (95\% CI 2.35-5.95), respectively, for successful deep sedation with a UMSS score $>3$ to start MRI in children with CP.

\section{References}

1. Truwit CL, Barkovich AJ, Koch TK, Ferriero DM. Cerebral palsy: MR findings in 40 patients. AJNR Am J Neuroradiol 1992; 13: 67-78.

2. Candy EJ, Hoon AH, Capute AJ, Bryan RN. MRI in motor delay: important adjunct to classification of cerebral palsy. Pediatr Neurol 1993; 9: 421-9.

3. Maxwell LG, Yaster M. The myth of conscious sedation. Arch Pediatr Adolesc Med 1996; 150: 665-7.
4. Hasan RA, Shayevitz JR, Patel V. Deep sedation with propofol for children undergoing ambulatory magnetic resonance imaging of the brain: experience from a pediatric intensive care unit. Pediatr Crit Care Med 2003; 4: 454-8.

5. Serafini G, Zadra N. Anaesthesia for MRI in the paediatric patient. Curr Opin Anaesthesiol 2008; 21: 499-503.

6. Sury MR, Smith JH. Deep sedation and minimal anesthesia. Paediatr Anaesth 2008; 18: 18-24.

7. Vangerven M, Van Hemelrijck J,Wouters P, Vandermeersch E, Van Aken H. Light anaesthesia with propofol for paediatric MRI. Anaesthesia 1992; 47: 706-7.

8. Frei FJ, Haemmerle MH, Brunner R, Kern C. Minimum alveolar concentration for halothane in children with cerebral palsy and severe mental retardation. Anaesthesia 1997; 52: 1056-60.

9. Saricaoglu F, Celebi N, Celik M, Aypar U. The evaluation of propofol dosage for anesthesia induction in children with cerebral palsy with bispectral index (BIS) monitoring. Paediatr Anaesth 2005; 15: 104852.

10. Dixon WJ. Quantal response to variable experimentation: the upand-down method. In: Statistics in endocrinology. Edited by Mc Arthur JW, Colton T: Cambridge, MIT Press. 1967, pp 251-64.

11. Paul M, Fisher DM. Are estimates of MAC reliable? Anesthesiology 2001; 95: 1362-70.

12. American Academy of Pediatrics; American Academy of Pediatric Dentistry, Cote CJ, Wilson S; Work Group on Sedation. Guidelines for monitoring and management of pediatric patients during and after sedation for diagnostic and therapeutic procedures: an up date. Pediatrics 2006; 118: 2587-602.

13. Cho JE, Kim WO, Chang DJ, Choi EM, Oh SY, Kil HK. Titrated propofol induction vs. continuous infusion in children undergoing magnetic resonance imaging. Acta Anaesthesiol Scand 2010; 54: 453-7.

14. Usher AG, Kearney RA, Tsui BC. Propofol total intravenous anesthesia for MRI in children. Paediatr Anaesth 2005; 15: 23-8.

15. Pace NL, Stylianou MP. Advances in and limitations of up-anddown methodology: a precise of clinical use, study design, and dose estimation in anesthesia research. Anesthesiology 2007; 107: 14452. 